\title{
Development of Artificial Neural Network for body composition Analysis
}

\author{
Nitin V Jadhav ${ }^{1}$, Prof. U.R.Bagal ${ }^{2}$ \\ ${ }^{1}$ (Biomedical dept., MGM CET, Kamothe, Navi Mumbai/ Mumbai University, India) \\ ${ }^{2}$ (Biomedical dept., MGM CET, Kamothe, Navi Mumbai/ Mumbai University, India)
}

\begin{abstract}
Artificial Neural networks (ANN) are finding many uses in the medical diagnosis applications. Different diseases such as acquired immuno deficiency syndrome (AIDS), malnutrition, cardiovascular diseases, osteoporosis are related to body composition topologies such as fat mass (FM), fat free mass (FFM), total body water $(T B W)$, bone mineral contents $(B M C)$ and bone mineral density $(B M D)$. Due to heterogeneous complexity of medical data classification and analysis needs Artificial Intelligence (AI) based technique to manipulate data. Many e-health system especially ANN uses AI methods to improve diagnostic process. Currently many body composition measurement systems in their applications elderly. Hence bioelectric impedance analysis (BIA) technique is used which is non-invasive, easy, fast and inexpensive. In biological structure, application of low level alternation of current produces impedance to spread current. These impedance and phage angle is measured using different electrodes to calculate resistive and reactive components of the body. These components along with other independent variables such as age, height, weight, etc are used to calculate BMC and BMD respectively. ANN provides mathematical equations which are used to calculate BMC and BMD, which are useful body composition parameters for the detection of osteoporosis.
\end{abstract}

Keywords: Artificial Neural network, acquired immuno deficiency syndrome, fat mass, fat free mass, bone mineral density, bone mineral contents, bone mineral density, artificial intelligence, bioelectric analysis, etc.

\section{Introduction}

The most important concern in the medical domain is to consider the interpretation of data and perform accurate diagnosis. A common disease 'Osteoporosis' does not depend on the bone mineral contents only but also some other significant factors such as age, height, weight, life style etc. All these factors play important role in a diagnosis of osteoporosis [1]. There are so many methods such as X-ray absorptiometry (DXA), hystrostatic weighing (HW), body mass index (BMI), and body impedance analysis (BIA) for classification of person to normal, osteopenia, osteoporosis, severe osteoporosis. Among them DXA and HW are expensive, time consuming, invasive, and not used in epidemiology studies. Therefore, many health systems have started using techniques e.g. Artificial Intelligence (AI) methods such as Artificial Neural Networks (ANN). It can provide highly accurate results in comparison with regression models. It Human body is consists of 50 to $60 \%$ of body fluid which functions as conductor at a high frequency. The living organisms behave as electrical conductor and cell membrane behaves as imperfect reactive elements.

In biological structures application of constant, low level current produces impedance to spread of current i.e. which is a frequency dependent. The body fluids and electrolytes are responsible for electric conductance and cell membrane is involved in reactance is a measurement of the ability of a medium to conduct current. It is the ratio of voltage (V) to the injected current (I). Phase angle $(\Phi)$ is the time delay between an alternating current in a conductive medium. It is expressed in degrees of phase shifts. Resistance (R) is a component of the impedance related to dissipation of energy in a conductive medium. Reactance $\left(\mathrm{X}_{\mathrm{c}}\right)$ is the components related to the storage of energy in a conductive medium [2]. Consider a cylinder having cross sectional area $A\left(\mathrm{~m}^{2}\right)$ and length $\mathrm{L}(\mathrm{m})$ of the uniform resistivity. Neural Network analysis is an outgrowth of AI. Neural Networks take a different approach to problem solving than that of conventional computers. In this paper perceptron algorithm was used to calculate output variables BMC and BMD using input variables such as age (years), height $(\mathrm{m})$, weight $(\mathrm{Kg})$, resistance $(\mathrm{R})$, and capacitance $\left(\mathrm{X}_{\mathrm{c}}\right)$. Mathematical equations were developed using input variables. $R$ and $X_{c}$ are calculated using formula in equations (2). From figure 1.1 impedance of the cylinder can be calculate can be calculated as,

$$
\begin{aligned}
& Z=\rho L / A \\
& \mathrm{R}=\mathrm{Z} \operatorname{Sin}(\varphi), \text { and } \mathrm{X}_{\mathrm{c}}=\mathrm{ZCOS}(\varphi), \\
& Z^{2}=R^{2}+X_{C}^{2}
\end{aligned}
$$




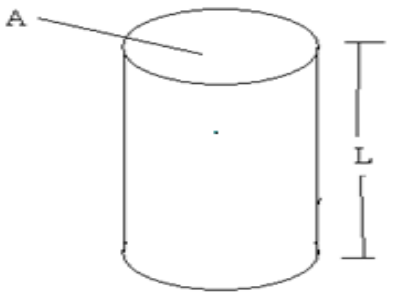

fig. 1.1 Cylinder with uniform resistivity

\section{Artificial Neural Network}

ANN behaves as a brain, and inspired by biological neural network (BNN). Neural network are a series of non-linear, interconnected mathematical equations, which resemble biological neuronal systems and are used to calculate an output variable on the basis of independent input variable. Neural Network analysis is an outgrowth of AI [3]. Neural Networks take a different approach to problem solving than that of conventional computers. In this paper, perceptron algorithm was used to calculate output variables BMC and BMD using input variables such as age (years), height $(\mathrm{m})$, weight $(\mathrm{Kg})$, resistance $(\mathrm{R})$, and capacitance $\left(\mathrm{X}_{\mathrm{c}}\right)$. Mathematical equations were developed using input variables. It consists of large number of simple processing elements that are interconnected with each other. It has three layers namely input layer, hidden layer and output layer. Input layer consists of different types of inputs which compute the total signal being sent to it by other processors in the network. Hidden layer consists of synaptic weights and bias that are applied to input variables present in input layers, the unit applies an activation function to this total signal to get desired. Output layer sends a signal to other processors. The way that the neurons are connected to each other has a significant impact on the operation of the ANN. It is trained to map a set of input data by iterative adjustment of the weights. Information from inputs is fed forward through the network to optimize the weights between neurons [3]. It learns and progressively develops meaningful \& reliable relationships between input and output variables. ANN can be used to solve a variety of problems in pattern recognition, prediction, and control systems. Conventional approaches have been proposed for solving these problems. ANN may be used to establish relationship between output parameters such as BMD, BMC and input parameters such as age, weight, height, gender (male/female), resistive and reactive component of human body. With their unique features neural network has become a powerful decision-making tool. Studies and investigations are being made to enhance the applications of ANNs and to achieve the benefits of this new technology [4]

\section{Feed Forward Network}

Generally, feed-forward networks each consist of three layers of artificial neurons. Data are entered in the input layer and further processed in the hidden and output layers. ANN use non-linear mathematical equations to successively develop meaningful relationships between input and output variables through a learning process, which consists of a "training phase" and a "recall phase".

\subsection{Training phase}

In this phase, the relationships between the different input variables and the output variable(s) are established through adaptations of the weight factors assigned to the interconnections between the layers of the artificial neurons. This adaptation is based on rules that are set in the learning algorithm. At the end of the training phase, the weight factors are fixed.

\subsection{Recall phase}

In this phase, data from patterns not previously interpreted by the network are entered, and an output is calculated based on the above-mentioned, and now fixed, weight factors. A layered feed-forward network consists of a certain number of layers, and each layer contains a certain number of units. A single-layer neuron is not able to learn and generalize the complex problems [6].

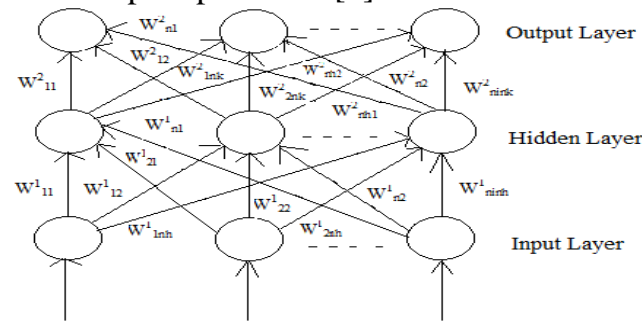

fig. 3.1 Feed forward network [5] 


\subsection{Perceptron algorithm}

Perceptrons architecture is a classical Neural Networks proposed by Frank Rosenblatt in 1957. The perceptron function is a classification of different patterns. A pattern can be considered as a point in $\mathrm{n}$ dimensional space (where $\mathrm{n}$ coordinates correspond to different features of the object to be classified). MLPs are the most common type of feed-forward networks. In the most common case, the perceptron was presented as a structure with one layer of neurons that are connected with inputs of the system. These connections have the weight coefficients, which can be changed during the training process. The goal is to find a set of weights w0, w1, wn such that the output of the perceptron is 1 if the input pattern vector belongs to class 1 and 0 if the pattern vector belongs to class 0 training. The weights are modified in accordance with the perceptron learning rule (or law). Neural Network was trained using supervised In other words, for each input X to the network, the correct output Y also was supplied [7].

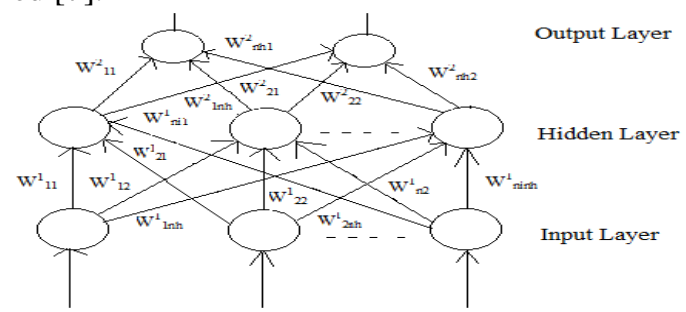

fig. 3.2 Multilayer Perceptron Network [5]

MLP is a feed forward neural network with one or more hidden layers. It is general function aproximator. MLP with one hidden layer as shown in Fig 3.2 is sufficient to approximate any continuous function. Input acts as input buffer data buffers that distribute the input to hidden layer. Hidden layer performs two functions i.e. combining function and activation function. Consider MLP with one hidden layer with $n_{i}$ input nodes, the output nodes of $\mathrm{j}^{\text {th }}$ neuron of hidden layer is given by,

$$
V_{j}=F\left(\left(\sum_{i=1}^{n} W^{1}{ }_{j i} x_{i}(t)+b j\right) ; \text { for } 1 \leq j \leq n_{h}\right.
$$

Where, the $\mathrm{W}^{1}{ }_{\mathrm{ji}}$ denotes the weight that connect the input and hidden layers; $\mathrm{x}_{\mathrm{i}}$, and $\mathrm{b}_{\mathrm{i}}$ denote the input that are supplied to the input layer and thresholds in hidden layers respectively. $\mathrm{n}_{\mathrm{i}}$ and $\mathrm{n}_{\mathrm{h}}$ are number of input and hidden nodes respectively. The output k-th output neuron, $\mathrm{y}_{\mathrm{k}}$ in the output layer is given by,

$$
y^{\wedge}{ }_{k}=\sum_{j=1}^{n h} W^{2}{ }_{k j} V_{j}(t) \text {; for } i \leq k=n_{o}
$$

Where $\mathrm{F}($.$) is activation function which may be sigmoid or hardlim activation function. The weights$ $\mathrm{W}_{\mathrm{ji}}^{1}, \mathrm{~W}^{2}{ }_{\mathrm{kj}}$ and threshold $\mathrm{b}_{\mathrm{j}}$ are unknown and should be selected to minimize the predication]n errors which is defined as

$$
\rho_{k}(t)=y^{\wedge} k(t)-y(t)
$$

Where $\hat{y}_{k}(t)=$ network output and $y_{k}(t)=$ obtained output.

\subsection{Transfer functions used in ANN}

Most units in neural network transform their net inputs by using a scalar-to-scalar function called an activation function, yielding a value called the unit's activation. Few of them have explained in the section 3.4.1, 3.4 2, 3.4.3, and 3.4.4

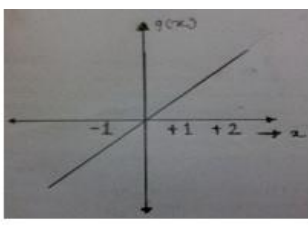

A) Identity function

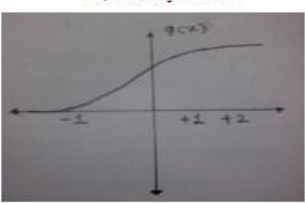

C) Sigmoid function

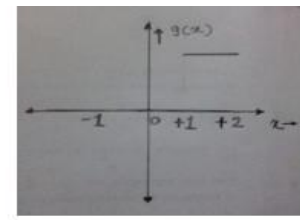

B) Binary step function

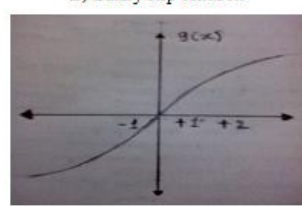

D) Bipolar sigmoid function

fig. 3.3 Activation functions used in ANN [19] 


\subsubsection{Identity function}

It is obvious that the input units use the identity function. Sometimes a constant is multiplied by the net input to form a linear function.

$$
g(x)=x
$$

Except possibly for the activation value is fed to one or more other units. Activation functions with a bounded range are often called squashing functions. [8].

\subsubsection{Binary step function}

This kind of function is often used in single layer networks. It is also known as threshold function or Heaviside function. The output of this function is limited to one of the two values:

$$
\begin{array}{ll}
g(x)=1 & \text { if } x \geq \theta \\
g(x)=0 & \text { if } x \prec \theta
\end{array}
$$

\subsubsection{Sigmoid function}

This function is especially advantageous for use in Neural Networks trained by back-propagation; because it is easy to differentiate, and thus can dramatically reduce the computation burden for training. It applies to applications whose desired output values are between 0 and 1 .

$$
g(x)=1 / 1+e^{-x}
$$

\subsubsection{Bipolar sigmoid function}

This function has similar properties with the sigmoid function. It works well for applications that yield output values in the range of $[-1,1]$. Activation functions for the hidden units are needed to introduce nonlinearity into the networks.

$$
g(x)=1-e^{-x} / 1+e^{-x}
$$

The reason is that a composition of linear functions is again a linear function. However, it is the nonlinearity (i.e., the capability to represent nonlinear functions) that makes multi-layer networks so powerful. Almost any nonlinear function does the job, although for back-propagation learning it must be differentiable and it helps if the function is bounded. The sigmoid functions are the most common choices [8]. For the output units, activation functions should be chosen to be suited to the distribution of the network output values. We have already seen that for binary $[0,1]$ outputs, the sigmoid function is an excellent choice. For continuous-valued network outputs with a bounded range, the sigmoid functions are again useful, provided that either the outputs or the network outputs to be scaled to the range of the output activation function. But if the network output values have no known bounded range, it is better to use an unbounded activation function, most often the identity function (which amounts to no activation function). If the network output values are positive but have no known upper bound, an exponential output activation function can be used [3].

\section{Development of an Artificial Neural Network for Body Composition Analysis} 4.1 Development of the Training Algorithms

For the development of Training algorithm of an ANN we need to following steps mentioned below:

\subsubsection{Access input-output data}

In order to train the ANN using supervised learning algorithm, input-output pairs or training data is required. This can be obtained easily by varying the inputs within the range for which training is required. Input parameters used in body composition analysis are age, weight, height, reactance and resistance etc. Resistance $(\mathrm{R})$, Reactance $\left(\mathrm{X}_{\mathrm{c}}\right)$ have been calculated using following formulae:

$$
\begin{aligned}
& \mathrm{R}=\mathrm{Z} \operatorname{Sin}(\varphi) \\
& \mathrm{X}_{\mathrm{c}}=\mathrm{Z} \operatorname{Cos}(\varphi)
\end{aligned}
$$

Where, Impedance $(\mathrm{Z})$ is a measurement of the ability of a medium to conduct current. It is the ratio of induced voltage $(\mathrm{V})$ to the injected current $(\mathrm{I})$. Phase angle $(\Phi)$ is the time delay between a stimulating current and voltage generated by alternating current in a conductive medium. It is expressed in degrees of phase shifts [1].

\subsubsection{Building a Network}

At this stage, the designer specifies the number of hidden layers, neurons in each layer transfer function in each layer, training function, weight/bias learning function, and performance function or activation function [9]. 


\subsubsection{Training of a network}

During the training process, the weights and bias are adjusted in order to make the actual outputs (predicated) close to the network output (measured) outputs of the network [4]. Network is trained to achieve desired results. Once it is developed correctly, it can be used for simulation purpose.

\subsubsection{Simulation of algorithm}

The network needs to be simulated and test input is applied after training of the network. The remaining input-output data is used to test the Network whether is perfectly trained or not. The output obtained and actual output has been plotted to check accuracy of a system.

\subsection{Method}

Our aim is to make a system which can calculate BMD and BMC using independent parameters such as Age, height, weight, sex (male/female), resistive and reactive components of body impedance. BMD and BMC have non linear relationship with all the above mentioned input parameters. Input parameters selected are age (in Year), sex (male/female), weight (in $\mathrm{Kg}$ ), and height (in meter), resistive and reactive components of the body which can be measured using equations (2) and (3) respectively ANN prediction equation were developed using impedances and other anthropometrics for predicting the reference BMC, BMD etc of 34 male and 82 female subjects. Out of which 28 males and 47 females were used for training purpose, and remaining data is used for simulation and testing the model. Many variables often have a high number of missing values, usually due to error or to the fact that the variable is measured only seldom. Provided enough data are available, the incomplete portion of the data can simply be discarded. However, the amount of training data is usually smaller than required. In order that even incomplete data can be used, many algorithms have been developed, as described in a previous comprehensive overview. Interestingly, again ANN can be used to approximate missing values. After suitable training, final weight and bias values are obtained which will give mathematical equations for BMD as well as BMC.

Following steps have to follow for perceptron algorithm for Body Composition Analysis:

Step 1: Access of input and output data.

Load datafile containing input parameters such as Age (X1), Height (X2), weight (X3), Reactive components(X4 \& X5) for BMD (T1) and BMC (T2) are taken into considerations.(The data shown in observation table is taken from [16] )

Step 2: Assumed some random Values of synaptic weight and bias.

Weight values and bias values and learning rate ('mu') and momentum ('alpha').Then, weighted inputs are summed together with bias. Multiply weights and input vectors.

$$
\begin{aligned}
& \sum W_{i} X_{i}=W_{1} X_{1}+W_{2} X_{2}+W_{3} X_{3}+W_{4} X_{4}+W_{5} X_{5} \\
& Y^{\wedge}{ }_{k}=\sum W_{i} X_{i}
\end{aligned}
$$

Step 3: Apply hardlim or sigmoid function to the output.

$$
y_{K}=F\left(Y_{k}^{\wedge}\right)
$$

Where $\mathrm{F}($.$) is a hardlim or sigmoid activation function$

Step 4: Calculation of error and updating weight and bias values.

$$
\rho_{k}(t)=y^{\wedge}(t)-y_{k}(t)
$$

Where, $\hat{y}_{\mathrm{k}}=$ network output $\mathrm{yk}=$ actual output

$$
\begin{aligned}
& W(\text { new })=\eta \rho(t) y_{k}+\mu W_{i j}(t) \\
& b(\text { new })=\eta \rho(t)+b_{i}(t)
\end{aligned}
$$

Step 5: Verify results with new weights and bias values.

Again compare output of bone mineral content with network output bone mineral content values and update weight and bias values if network output does not matches with output. Keep maximum no of iteration get maximum accuracy.

Step 6: Finalized weight and bias values after suitable training.

We obtain fixed values of weights and bias.

Step 7: Same procedure is followed for BMD as well as BMC also. (Follow step 1 to 6). The perceptron architecture network is developed and trained successfully. 


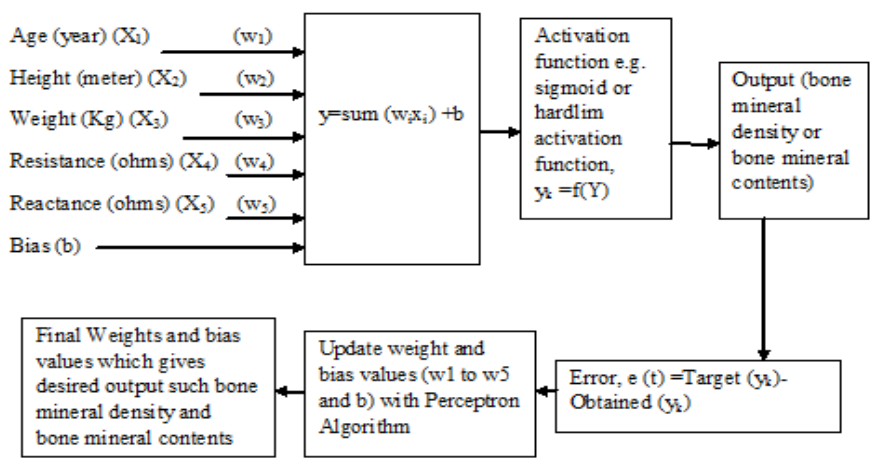

fig 4.1 Block diagram BIA for determination of bone mineral density and Bone mineral contents

\section{Indentations and EQUATIONS}

Injection of a frequency dependent current (about $1 \mathrm{~mA}$ ) produces an impedance $(\Omega)$ and phase angle $(\Phi)$ which was used to calculate resistance $(\Omega)$ and reactive components $(\Omega)$ of human body. Single layer perceptron model was trained successfully using input and target output or output parameters. Input parameters used for development of algorithm were age (year), height (meter), weight $(\mathrm{Kg})$, resistance $(\Omega)$, and reactance $(\Omega)$ while respective output parameters are BMD and BMC. Following equations shows the relation between input and output parameters mentioned above.

$$
\begin{gathered}
B M D(\text { male })=0.0094+0.0088 \text { Age }+0.0013 \text { Height }+0.0095 \text { Weight }-0.0036 R-0.0008 X c \\
B M C(\text { male })=0.0074+0.0266 \text { Age }+0.0069 \text { Height }+0.01595 \text { Weight }-0.0072 R-0.0306 X c \\
B M C(\text { female })=0.000562+0.0157 \text { Age }+0.0081 \text { Height }+0.0181 \text { Weight }+0.0123 R+0.0075 X c \\
B M D(\text { female })=0.00046+0.0084 \text { Age }+0.1983 \text { Height }+0.0079 \text { Weight }-0.0084 R-0.0043 X c
\end{gathered}
$$

\section{Figures and TABLES}

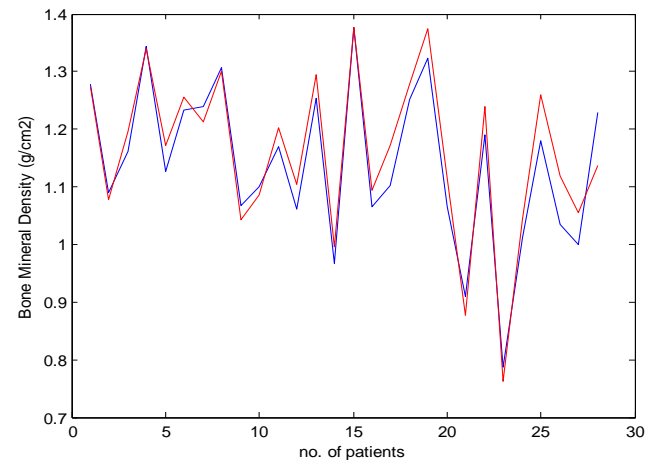

fig 6.1 Bone mineral density $\left(\mathrm{g} / \mathrm{cm}^{3}\right)$ for males

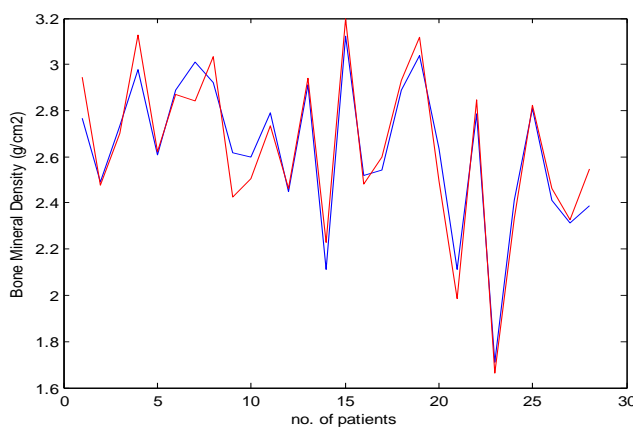

fig 6.3 Bone mineral contents $(\mathrm{Kg})$ for males

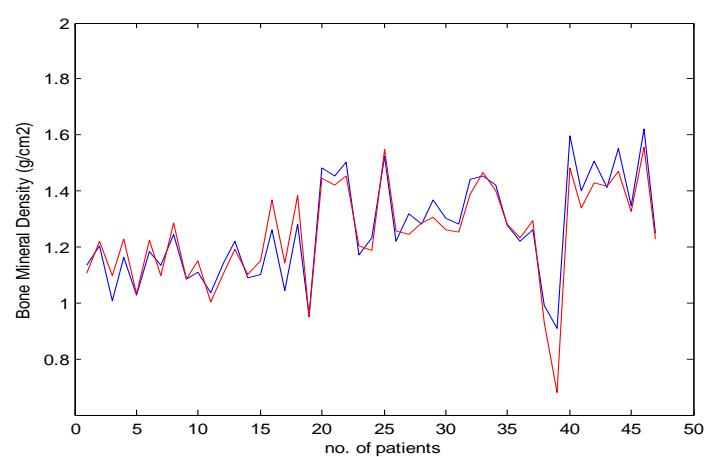

fig 6.2 Bone mineral density $\left(\mathrm{g} / \mathrm{cm}^{3}\right)$ for females

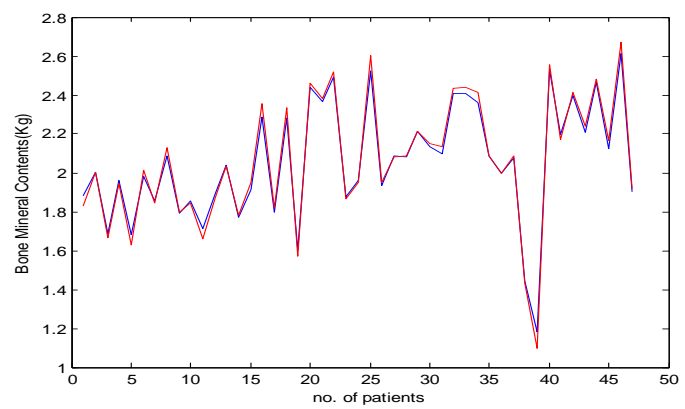

fig 6.4 Bone mineral density $(\mathrm{Kg})$ for females 
Observation Table -6.1 Bone mineral contents and bone mineral density data for 116 subjects

\begin{tabular}{|c|c|c|c|c|c|}
\hline $\begin{array}{l}\text { S } \\
\text { N }\end{array}$ & $\mathbf{M} / \mathbf{F}$ & $\begin{array}{c}\text { Age } \\
\text { (year) }\end{array}$ & $\begin{array}{c}\text { Height } \\
\text { (m) }\end{array}$ & $\begin{array}{c}\text { Weight } \\
\text { (Kg) }\end{array}$ & $\begin{array}{l}\text { Impedance } \\
\text { (ohms) }\end{array}$ \\
\hline & M & 64 & 1.685 & 73.2 & 1428.137 \\
\hline 2 & M & 54 & 1.73 & 61 & 1397.246 \\
\hline 3 & M & 49 & 1.69 & 79.2 & 1224.519 \\
\hline 4 & M & 72 & 1.68 & 73.1 & 1079.383 \\
\hline & M & 45 & 1.88 & 79.8 & 1217.75 \\
\hline 6 & M & 57 & 1.735 & 78.1 & 1162.583 \\
\hline 7 & M & 69 & 1.67 & 61.7 & 1263.417 \\
\hline 8 & M & 70 & 1.75 & 70.5 & 1054.969 \\
\hline 9 & M & 57 & 1.65 & 54.6 & 1271.979 \\
\hline 10 & M & 56 & 1.64 & 60.1 & 1352.191 \\
\hline 11 & M & 52 & 1.625 & 77.3 & 1057.479 \\
\hline 12 & M & 41 & 1.77 & 76.5 & 198.377 \\
\hline 13 & $\mathrm{M}$ & 55 & 1.715 & 84.4 & 1076.646 \\
\hline 14 & M & 39 & 1.66 & 66.7 & 1176.649 \\
\hline 15 & M & 71 & 1.72 & 78 & 1225.27 \\
\hline 16 & M & 47 & 1.57 & 70.1 & 1039.349 \\
\hline 17 & M & 39 & 1.73 & 86.1 & 1051.65 \\
\hline 18 & M & 59 & 1.78 & 78. & 22 \\
\hline 19 & M & 57 & 1.76 & 91.7 & 1082.675 \\
\hline 20 & M & 42 & 1.69 & 77.1 & 1047.969 \\
\hline 21 & M & 40 & 1.565 & 52.7 & 1305.87 \\
\hline 22 & M & 58 & 1.67 & 75.7 & 953.5547 \\
\hline 23 & M & 43 & 1.72 & 78.4 & 1052.877 \\
\hline 24 & M & 25 & 1.67 & 54. & 139 \\
\hline 25 & M & 38 & 1.59 & 69.2 & 1057.465 \\
\hline 26 & M & 45 & 1.62 & 73.4 & 1075.078 \\
\hline 27 & M & 32 & 1.745 & 88.3 & 1035.144 \\
\hline 28 & M & 35 & 1.76 & 76.8 & 1150.84 \\
\hline 29 & M & 40 & 1.52 & 48.5 & 1435.155 \\
\hline 30 & M & 66 & 1,53 & 718 & \\
\hline 31 & M & 54 & 1.5 & 84. & 127 \\
\hline 32 & M & 68 & 1.56 & 56.6 & 1817.259 \\
\hline 33 & M & 40 & 1.52 & 48.5 & 1435.155 \\
\hline 34 & M & 66 & 1.53 & 71.8 & 1320.155 \\
\hline 35 & M & 54 & 1.55 & 84.5 & 127 \\
\hline 36 & $\mathrm{M}$ & 68 & 1.56 & 56.6 & 1817 \\
\hline 37 & $\mathrm{~F}$ & 49 & 1.65 & 55.6 & 1656.782 \\
\hline 38 & F & 59 & 1.512 & 81.3 & 1336.268 \\
\hline 39 & F & 48 & 1.5156 & 65.5 & 1358.42 \\
\hline 40 & F & 66 & 1.525 & 54.9 & 1403.49 \\
\hline 41 & $\mathrm{~F}$ & 62 & 1 & 65 & 1367 \\
\hline 42 & F & 59 & 1.575 & 76.5 & 346.591 \\
\hline 43 & F & 38 & 1. & 70 & 36 \\
\hline 44 & $\mathrm{~F}$ & 58 & 1.6 & 85.4 & 1303.697 \\
\hline 45 & $\mathrm{~F}$ & 64 & 1.56 & 75.3 & 1346.185 \\
\hline 46 & F & 24 & 1.58 & 53.5 & 1821.5 \\
\hline 47 & F & 63 & 1.4 & 43.2 & 1720.476 \\
\hline 48 & F & 54 & 1.525 & & 762 \\
\hline 49 & $F_{1}$ & 43 & 1.6 & & \\
\hline 50 & F & 34 & 1.5 & .3 & 1063 \\
\hline 51 & & 47 & 1.655 & 62.2 & 1753.68 \\
\hline & $\mathrm{F}$ & 55.6 & 1.46 & 55.6 & 1327.29 \\
\hline & & 58 & 1.6 & & 1065 \\
\hline & & 59 & 1.5 & 48 & \\
\hline 5 & & 2 & 1. & & \\
\hline 36 & F & 60 & 1.48 & 58.9 & 13000.25 \\
\hline 57 & F & 66 & 1.47 & 56.8 & 1364.871 \\
\hline 58 & F & 55 & 1.555 & 63.3 & 1365.487 \\
\hline
\end{tabular}

$\begin{array}{ccc}\begin{array}{c}\text { Phase } \\ \text { angle } \\ \text { (degree) }\end{array} & \begin{array}{c}\text { Bone } \\ \text { mineral } \\ \text { content } \\ \text { (Kg) }\end{array} & \begin{array}{c}\text { Bone mineral } \\ \text { density } \\ (\mathbf{g} / \mathbf{c m} \text { ) }\end{array} \\ 4.892 & 2.327 & 1.052 \\ 5.096 & 2.492 & 1.125 \\ 6.405 & 2.953 & 1.279 \\ 5.294 & 2.584 & 1.163 \\ 4.8 & 3.031 & 1.168 \\ 5.32 & 2.911 & 1.208 \\ 4.458 & 2.456 & 1.118 \\ 5.657 & 2.625 & 1.101 \\ 4.5 & 2.452 & 1.174 \\ 4.595 & 2.707 & 1.226 \\ 5.263 & 2.505 & 1.161 \\ 5.3 & 2.866 & 1.092 \\ 5.559 & 2.559 & 1.084 \\ 6.098 & 2.648 & 1.182 \\ 4.339 & 2.466 & 1.074 \\ 5.837 & 2.314 & 1.154 \\ 4.499 & 2.83 & 1.038 \\ 5.608 & 2.706 & 1.162 \\ 4.535 & 3.03 & 1.191 \\ 5.75 & 2.634 & 1.145 \\ 6.771 & 2.113 & 1.086 \\ 5.181 & 2.768 & 1.139 \\ 6.692 & 2.318 & 1.098 \\ 5.964 & 2.285 & 1.105 \\ 6.031 & 2.48 & 1.239 \\ 5.578 & 2.866 & 1.287 \\ 5.866 & 2.709 & 1.141 \\ 5.925 & 2.998 & 1.199 \\ 4.997 & 1.597 & 1.131 \\ 4.79 & 2.43 & 1.554 \\ 4.779 & 2.52 & 1.56 \\ 4.903 & 2.231 & 1.54 \\ 4.993 & 1.79 & 1.69 \\ 4.79 & 2.73 & 1.31 \\ 4.779 & 2.51 & 1.44 \\ 4.902 & 2.653 & 1.14 \\ 5.035 & 2.042 & 1.011 \\ 4.64 & 1.956 & 1.214 \\ 5.626 & 2.19 & 1.116 \\ 5.032 & 1.703 & 0.922 \\ 4.392 & 1.862 & 0.939 \\ 3.449 & 1.959 & 0.968 \\ 5.744 & 2.69 & 1.193 \\ 4.756 & 2.13 & 0.973 \\ 4.99 & 2.017 & 1.051 \\ 5.763 & 2.166 & 1.019 \\ 3.586 & 1.326 & 0.943 \\ 4.497 & 1.823 & 1.018 \\ 4.474 & 1.958 & 1.044 \\ 5.565 & 2.72 & 1.222 \\ 4.543 & 2.317 & 1.186 \\ 4.334 & 2.01 & 1.114 \\ 5.362 & 2.478 & 1.199 \\ 4.842 & 1.416 & 0.876 \\ 4.831 & 2.196 & 1.109 \\ 4.456 & 2.048 & 1.091 \\ 4.421 & 1.882 & 1.161 \\ 5.134 & 2.226 & 1.112 \\ & & \\ & & \end{array}$

\begin{tabular}{|c|c|c|c|c|c|c|c|c|}
\hline $\begin{array}{l}\text { Sr. } \\
\text { No }\end{array}$ & $\mathrm{M} / \mathrm{F}$ & $\begin{array}{c}\text { Age } \\
\text { (year) }\end{array}$ & $\begin{array}{c}\text { Height } \\
(\mathrm{m})\end{array}$ & $\begin{array}{l}\text { Weight } \\
\text { (Kg) }\end{array}$ & $\begin{array}{c}\text { Impedance } \\
\text { (ohms) }\end{array}$ & $\begin{array}{c}\text { Phase } \\
\text { angle } \\
\text { (degree) }\end{array}$ & $\begin{array}{l}\text { munc } \\
\text { mineral } \\
\text { content } \\
(\mathrm{K} \sigma)\end{array}$ & $\begin{array}{l}\text { Bone mineral } \\
\text { density } \\
(\mathrm{g} / \mathrm{cm} 2)\end{array}$ \\
\hline 59 & $\mathrm{~F}$ & 68 & 1.635 & 89.9 & 1399.89 & 4.511 & 2.11 & 1.086 \\
\hline 60 & F & 70 & 1.51 & 69.5 & 1333.244 & 2.812 & 1.503 & 0.857 \\
\hline 61 & $\mathrm{~F}$ & 58 & 1.555 & 68.4 & 1299.578 & 4.789 & 2.095 & 1.093 \\
\hline 62 & $\mathrm{~F}$ & 45 & 1.555 & 47.7 & 1703.985 & 4.348 & 1.872 & 1.005 \\
\hline 63 & $\mathrm{~F}$ & 48 & 1.63 & 63.3 & 1510.531 & 4.671 & 2.072 & 1.031 \\
\hline 64 & $\mathrm{~F}$ & 49 & 1.525 & 54.7 & 1574.672 & 4.108 & 1.838 & 0.984 \\
\hline 65 & $\mathrm{~F}$ & 67 & 1.53 & 46.6 & 1649.573 & 3.848 & 1.46 & 0.931 \\
\hline 66 & $\mathrm{~F}$ & 64 & 1.64 & 63.8 & 1513.483 & 4.415 & 2.455 & 1.161 \\
\hline 67 & $\mathrm{~F}$ & 49 & 1.61 & 68.8 & 1499.642 & 4.747 & 2.102 & 1.056 \\
\hline 68 & $\mathrm{~F}$ & 61 & 1.61 & 84.1 & 13000.987 & 4.726 & 2.259 & 1.138 \\
\hline 69 & $\mathrm{~F}$ & 46 & 1.48 & 53.9 & 1541.188 & 4.246 & 1.825 & 1.012 \\
\hline 70 & $\mathrm{~F}$ & 70 & 1.45 & 76.1 & 1279.857 & 4.481 & 1.718 & 0.973 \\
\hline 71 & $\mathrm{~F}$ & 66 & 1.605 & 58.1 & 1702.073 & 3.611 & 1.46 & 0.866 \\
\hline 72 & $\mathrm{~F}$ & 45 & 1.575 & 59.1 & 1372.252 & 4.74 & 2.353 & 1.118 \\
\hline 73 & $\mathrm{~F}$ & 45 & 1.665 & 49.3 & 1628.384 & 4.138 & 2.204 & 1.14 \\
\hline 74 & $\mathrm{~F}$ & 48 & 1.68 & 57.4 & $\begin{array}{l}1593.972 \\
\end{array}$ & $\begin{array}{l}4.786 \\
4.786\end{array}$ & 2.54 & 1.125 \\
\hline 75 & $\mathrm{~F}$ & 39 & 1.59 & 61.4 & 1445.732 & 4.908 & 2.214 & 1.099 \\
\hline 76 & $\mathrm{~F}$ & 54 & 1.65 & 66.6 & 1568.176 & 3.92 & 1.976 & 1.002 \\
\hline 77 & $\mathrm{~F}$ & 52 & 1.53 & 67.5 & 1194.127 & 4.428 & 2.158 & 1.113 \\
\hline 78 & F & 51 & 1.59 & 59.1 & 1560.575 & 4.292 & 2.289 & 1.106 \\
\hline 79 & $\mathrm{~F}$ & 48 & 1.59 & 71.3 & 1282.884 & 4.914 & 2.675 & 1.203 \\
\hline so & $\mathrm{F}$ & 78 & 1.535 & 43.7 & 1348.472 & 3.759 & 1.701 & 0.945 \\
\hline 81 & $\mathrm{~F}$ & 40 & 1.52 & 48.5 & $\begin{array}{l}134.452 \\
1435.155\end{array}$ & $\begin{array}{l}4.997 \\
4.997\end{array}$ & 2.123 & 1.174 \\
\hline 82 & $\mathrm{~F}$ & 66 & 1.53 & 71.8 & 1320. & 4.79 & 2.085 & 1.054 \\
\hline 83 & $\mathrm{~F}$ & 54 & 1.55 & 84.5 & 1277. & 4.779 & 1.9 & $\begin{array}{l}1.038 \\
\text {. }\end{array}$ \\
\hline 84 & $\mathrm{~F}$ & $\begin{array}{l}64 \\
68\end{array}$ & 1.56 & 56.6 & $\begin{array}{l}1817.259 \\
\end{array}$ & $\begin{array}{l}4.902 \\
4.902\end{array}$ & $\begin{array}{l}1.751 \\
1.751\end{array}$ & $\begin{array}{l}1.003 \\
1.003\end{array}$ \\
\hline 85 & $\mathrm{~F}$ & 75 & 1.65 & 69.7 & 1306.352 & 3.594 & 2.25 & 1.033 \\
\hline 86 & $\mathrm{~F}$ & 49 & 1.56 & 49.6 & 1527.646 & 4.479 & 1.7914 & 0.98 \\
\hline $\begin{array}{l}80 \\
87\end{array}$ & F & 74 & $\begin{array}{l}1.40 \\
1.46\end{array}$ & $\begin{array}{l}49.0 \\
45\end{array}$ & $\begin{array}{l}172.70406 \\
1738.164\end{array}$ & $\begin{array}{l}4.449 \\
4.517\end{array}$ & 1.93 & $\begin{array}{l}1.011 \\
1.011\end{array}$ \\
\hline 88 & $\mathrm{~F}$ & 57 & 1.57 & 80.9 & 1383 . & 4.227 & 2.1 & 1.283 \\
\hline 89 & $\mathrm{~F}$ & 69 & 1.56 & 62.2 & 1405.974 & 4.716 & 1.81 & 0.976 \\
\hline 90 & $\mathrm{~F}$ & 57 & $\begin{array}{l}1.67 \\
\end{array}$ & 81.3 & $\begin{array}{l}1192.065 \\
1195\end{array}$ & 4.556 & 2.28 & 1.2143 \\
\hline 91 & F & 54 & 1.56 & 64.3 & 1364.366 & 4.321 & 2.221 & 1.12 \\
\hline 92 & $\mathrm{~F}$ & 67 & 1.56 & 72.9 & 1316.393 & 4.675 & 2.197 & 1.31 \\
\hline 93 & $\mathrm{~F}$ & 51 & 1.55 & 62 & 1364.971 & 5.023 & 1.968 & 1.149 \\
\hline 94 & $\mathrm{~F}$ & 40 & 1.56 & 89.9 & 1271.363 & 4.905 & 2.444 & 1.244 \\
\hline 95 & $\mathrm{~F}$ & 64 & 1.56 & 87.8 & 974.4207 & 4.32 & 2.039 & 1.178 \\
\hline 96 & $\mathrm{~F}$ & 53 & 1.61 & 64.7 & 1385.747 & 5.786 & 2.265 & 1.058 \\
\hline 97 & $\mathrm{~F}$ & 63 & 1.5 & 57.5 & 1507.701 & 4.962 & 1.885 & 1.06 \\
\hline 98 & $\mathrm{~F}$ & 65 & 1.55 & 41 & 1858.888 & 4.264 & 1.556 & 0.949 \\
\hline 99 & $\mathrm{~F}$ & 40 & 1.55 & 48.9 & 1685.517 & 5.683 & 1.732 & 1.097 \\
\hline 100 & $\mathrm{~F}$ & 72 & 1.55 & 58.5 & 1424.049 & 3.237 & 1.575 & 0.869 \\
\hline 101 & F & 62 & 1.55 & 61.1 & $\begin{array}{l}1425.4049 \\
1558.666\end{array}$ & 3.825 & $\begin{array}{l}1.763 \\
1.763\end{array}$ & 0.976 \\
\hline 102 & $\mathrm{~F}$ & 36 & 1.57 & 66.5 & 1548.391 & 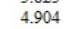 & 1.9 & 1.114 \\
\hline $\begin{array}{l}103 \\
103\end{array}$ & $\mathrm{~F}$ & 48 & 1.5 & 77.7 & 1137. & 5.3 & 2.1 & 1.22 \\
\hline 104 & F & 60 & $\begin{array}{l}1.5 \\
1.5\end{array}$ & 64.3 & 14 & 4.6 & 2.2 & 1.122 \\
\hline 105 & $\mathrm{~F}$ & 50 & 1.59 & 60.2 & 1370.357 & 5.083 & 2.247 & 1.22 \\
\hline 106 & $\mathrm{~F}$ & 64 & 1.61 & 97.7 & 1154.924 & 4.539 & 2.11 & 1.122 \\
\hline $\begin{array}{l}107 \\
107\end{array}$ & $\mathrm{~F}$ & $\begin{array}{l}84 \\
49\end{array}$ & 1.54 & 64.4 & 1313.437 & $\begin{array}{l}4.358 \\
4.388\end{array}$ & 2.223 & $\begin{array}{l}1.1142 \\
1.142\end{array}$ \\
\hline 108 & $\mathrm{~F}$ & 61 & 1.54 & 86.8 & 1038. & 4.002 & 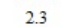 & 1.141 \\
\hline 109 & $\mathrm{~F}$ & 53 & 1.6 & 64.8 & 1414 & 3.9 & & 1.73 \\
\hline 110 & $\mathrm{~F}$ & 81 & 1.54 & 75.9 & 1261.777 & 5.061 & 2.059 & 0.907 \\
\hline 111 & $\mathrm{~F}$ & 66 & 1.52 & 66.6 & 1305.879 & 4.181 & 2.101 & 1.131 \\
\hline $\begin{array}{l}111 \\
112\end{array}$ & $\begin{array}{l}F \\
F\end{array}$ & $\begin{array}{l}00 \\
77\end{array}$ & $\begin{array}{l}1.22 \\
1.47\end{array}$ & $\begin{array}{l}0.0 \\
45\end{array}$ & $\begin{array}{l}150.86 .668 \\
1516.06\end{array}$ & $\begin{array}{l}4.181 \\
3.27\end{array}$ & 2.167 & $\begin{array}{l}1.131 \\
1.0341\end{array}$ \\
\hline 113 & $\mathrm{~F}$ & 51 & $\begin{array}{l}1.57 \\
1.57\end{array}$ & 64 & $\begin{array}{l}1311.079\end{array}$ & 4.458 & 2.14 & 1.174 \\
\hline 114 & $\mathrm{~F}$ & 44.4 & 1. & 71.1 & & 4. & 2.1 & 1. \\
\hline $\begin{array}{l}114 \\
115\end{array}$ & F & $\begin{array}{l}4.44 \\
32\end{array}$ & 1.63 & $\begin{array}{l}11.1 \\
64.8\end{array}$ & 1388.277 & $\begin{array}{l}4.403 \\
5.083\end{array}$ & 2.373 & $\begin{array}{l}1.157 \\
1.157\end{array}$ \\
\hline $\begin{array}{l}113 \\
116\end{array}$ & $\begin{array}{l}F \\
F\end{array}$ & $\begin{array}{l}32 \\
42\end{array}$ & $\begin{array}{l}1.03 \\
1.51\end{array}$ & $\begin{array}{l}64.8 \\
67.4\end{array}$ & 1280.18 & $\begin{array}{l}100.8 \\
100.8\end{array}$ & $\begin{array}{l}2.375 \\
2.433\end{array}$ & $\begin{array}{l}1.1197 \\
1.192\end{array}$ \\
\hline
\end{tabular}

\section{Conclusion}

The Perceptron network is most often used in the medical diagnosis systems. ANN analysis is performed to estimate the BMD as well as BMC. The BMD is the most accurate indicator of the risk factor for fracture, osteopenia, and osteoporosis. Perceptron model is developed and provides 90 to $95 \%$ accurate results which are shown in observation table as well as discriminant analysis of target output and obtained outputs for BMD and BMC. 6.1, 6.2, 6.3, 6.4 blue colours indicate target output values while red colour indicates obtained values. This application allows us to make non-linear relationship with input and output parameters of body composition analysis. The attributes taken for diagnoses are; Age (months), Sex (male/female), Height (inch), weight $(\mathrm{kg})$, Resistive as well as reactive components of body, BMD (g/cm), BMC $(\mathrm{Kg})$, Reactance $(\Omega)$, Capacitance $(\Omega)$ etc.

\section{Acknowledgements}

I am GRATEFUL to Mahatma Gandhi Mission (MGM) Trust and Dr. S K Narayankhedekar, Principal, MGM College of Engineering and Technology, and G.D. Jindal, Head of the dept. for giving me an opportunity to carry out my dissertation work. I express my extreme gratitude to my guide Prof. U. R. Bagal for guiding me throughout the work. I have been greatly benefited by his valuable suggestions and ideas. Without his guidance this work would not have materialized. I am also grateful to Prof. Aarti Bokade, Prof Bipin Mokal for providing valuable help from time to time.

[1] Abdul Basit Shaikh et.al, "Artificial Neural Network: A Tool for Diagnosing Osteoporosis”, RJRS, vol. 3(2), pp. 87-91, 2014, [online] Available: www.isca.in

[2] Psaltis, D., A. Sideris et.al, “A Multilayered Neural Network Controller,” IEEE Control Systems Magazine, vol. 8, No. 2, pp. 1721,1988

[3] Xiong Xin et.al. "BP Neural Network principle and MATLAB Simulation", pp. 1-6, Wuhan University, China

[4] Vidushi Sharma et al., "A Comprehensive Study of Artificial Neural Networks," IJARCSSE, vol.2, no. 10, pp. 278-284, 2012 
[5] Manaswamini Pradhan and Ranjit Kumar Sahu, "Multilayer Perceptron Network in HIV/AIDS Applications” IJCAES, vol-1, pp. 41-48, 2011

[6] K. Hornik, M. Stinchcombe et. al, "Multilayer feed forward networks are universal approximators," NN, vol. 2, no. 5,pp. 359366, 1989

[7] S N Sivanandam et al., "Introduction to Artificial Neural Networks," in Introduction To Neural Networks Using MATLAB 6.0, New Delhi, India: Tata McGraw-Hill, 2006. ch. 2, pp. 10-29

[8] Manuel Laguna et.al, "Neural Network prediction in a systems for optimizing simulations", IIE Transactions, vol. 34, pp. 273282, Colorado University, 2001

[9] Kuen-Chang Hsieh et al., "The novel application of artificial neural network on bioelectrical impedance analysis to assess the body composition in elderly," Nutrition Journal, vol. 12, no. 21, pp. 1-8, 2013

[10] B.R.Patil "Estimation of bone mineral content from bioelectric impedance analysis in Indian adults aged 23-81years: Comparison with dual energy X-ray absorptiometry" under review in the IJOI, IOF and the NOF, USA

[11] R. Linder et al., "The Capabilities of artificial neural networks in body composition research," unpublished

[12] Meeuwsen S,et.al., The relationship between BMI and percent body fat, measured by bioelectrical impedance, in a large adult sample is curvilinear and influenced by age and sex., Clinical Nutrition, vol.29 pp. 560-566. 2010

[13] Qeethara Kadhim Al-Shayea, "Artificial Neural Networks in Medical Diagnosis”, IJCSI, vol. 8, no. 2, pp. 150-154, 2011

[14] Irfan Y. Khan et.al., "Importance of Artificial Neural Network in Medical Diagnosis disease like acute nephritis disease and heart disease," IJESIT, vol. 2, no. 2, pp. 210-217, 2001

[15] Anil Jain “Artificial Neural Networks-A tutorial," IEEE, Almaden, Michigan, vol, 18, pp. 31-45, 1996

[16] B.R. Patil et.al, "Estimation of bone mineral content from bioelectric impedance analysis in Indian adults aged 23-81years: Comparison with dual energy X-ray absorptiometry", vol.8, no.1, pp.99-114, IJBET, 2012 